Provided for non-commercial research and education use. Not for reproduction, distribution or commercial use.

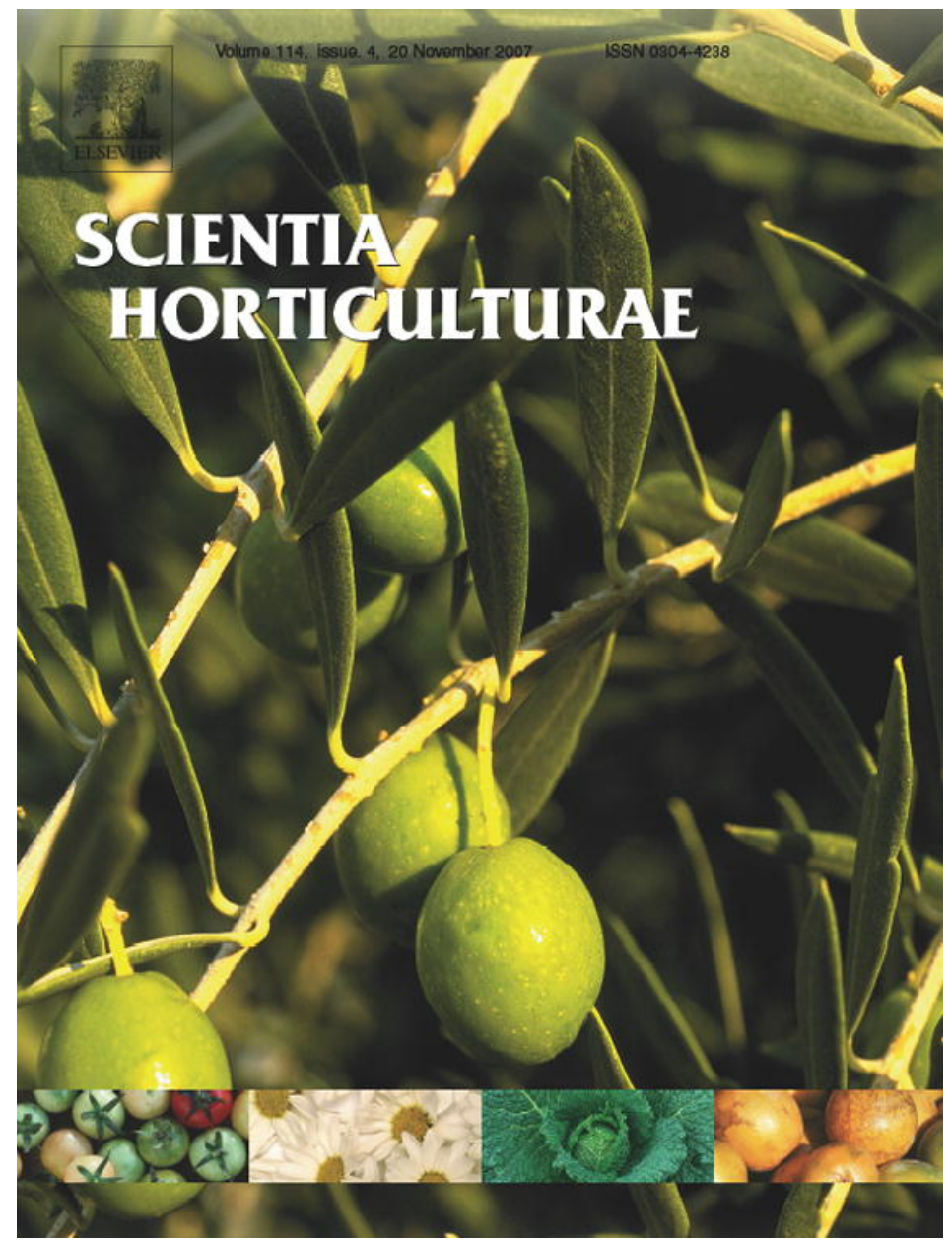

This article was published in an Elsevier journal. The attached copy

is furnished to the author for non-commercial research and education use, including for instruction at the author's institution, sharing with colleagues and providing to institution administration.

Other uses, including reproduction and distribution, or selling or licensing copies, or posting to personal, institutional or third party websites are prohibited.

In most cases authors are permitted to post their version of the article (e.g. in Word or Tex form) to their personal website or institutional repository. Authors requiring further information regarding Elsevier's archiving and manuscript policies are encouraged to visit: 


\title{
Evaluation of fruit and seed diversity and characterization of carob (Ceratonia siliqua L.) cultivars in Algarve region
}

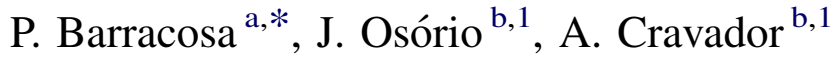 \\ ${ }^{a}$ Departamento de Produção Vegetal, Escola Superior Agrária de Viseu - ISPV, Quinta da Alagoa - Ranhados, 3500-Viseu, Portugal \\ ${ }^{\mathrm{b}}$ Universidade do Algarve-FERN, Campus de Gambelas, 8005-139 Faro, Portugal \\ Received 27 July 2006; received in revised form 21 June 2007; accepted 26 June 2007
}

\begin{abstract}
The genetic diversity of 15 carob (Ceratonia siliqua L.) cultivars located in an experimental field from Algarve (Portugal) was evaluated over 7 years using 12 fruit and seed phenotypic characters, in order to characterize carob cultivars. The values of morphological traits obtained by cultivar were compared with those from other countries of the Mediterranean basin. Statistically significant differences were found between cultivars for all characters which were examined, what indicates a high genetic diversity. The relationship among these characters was analyzed by principal component analysis (PCA) resulting in the separation of these cultivars classed in four groups (clusters I-IV) and in four ungrouped cultivars. A three dimension of the model was found to be significant and explained $74.5 \%$ of the total variation, in which the first component accounting for $34.6 \%$ of the total variation is dominated by fruit characters, while the second component is dominated by seed characters. Cultivars plotted on the left-lower quadrant on the space determined by principal components 1 and 2 are characterized by fruits with high seed yield more appropriated for industrial rentability. The correlation analyses established by cultivar provided a specific understanding about the way how fruit and seed characteristics correlate within each cultivar. This approach can be useful for the development of a breeding programme, aiming to increase the seed yield, seed thickness, individual and total seed weight by fruit, characteristics that are determinant to improve the industrial exploitation of carob.
\end{abstract}

(C) 2007 Published by Elsevier B.V.

Keywords: Carob; Cultivars; Morphological diversity; Principal component analysis; Correlation analysis

\section{Introduction}

Carob (Ceratonia siliqua L.; $2 n=24$ ) a leguminous subtropical species that belongs to the Leguminosae family, Caesalpinioidea sub-family, is a characteristic constituent of the evergreen, maquis-type vegetation in low-elevation areas in the Mediterranean basin. The genus Ceratonia L. has been assumed for long time to consist only of $C$. siliqua L. until the discovery of the new species Ceratonia oreothauma Hillcoat, Lewis and Verdc.sp. nov., from Oman and the Somali Republic (Hillcoat et al., 1980).

Taxonomically, Ceratonia is regarded as one of the most archaic of the legume genera (Tucker, 1992), completely

\footnotetext{
* Corresponding author. Tel.: +351 232 480600; fax: +351 232426536.

E-mail addresses: pbarracosa@esav.ipv.pt (P. Barracosa), josorio@ualg.pt (J. Osório), acravad@ualg.pt (A. Cravador).

${ }^{1}$ Tel.: +351289 800935; fax: +351289818419.
}

isolated from all other genera of its family (Zohary, 1973). It has been considered as originating from xerotropical IndoMalesian flora, grouping with Olea, Laurus, Myrtus, Chamaerops; the origin of its genus has also been placed on the Arabian Peninsula (Zohary, 1973).

Zohary (1973) suggested that the Mediterranean region has been one of its domestication centres and carob was brought into cultivation relatively later with the "second wave" of fruit crops domesticated in the Old World. Since early ages, the domestication of $C$. siliqua based on spontaneous populations, aiming at the production of large fruits with high sugar content for human and animal nutrition resulted in a limited number of cultivars (Zohary, 1983; Mitrakos, 1987). More recently, domestication of some wild uncultivated trees has been developed with the purpose of increasing seed yield and gum quality for industrial exploitation.

Pods and seeds of this species are used as row material in food, pharmaceutical and cosmetic industries (Carlson, 1986). The most widely used carob product in the food industry is the 
Locust Bean Gum (LBG), which comes from the endosperm of the seed, and is classified chemically as a galactomannan, widely used as a natural food additive (E 410). LBG has found increasing applications in the pharmaceutics industry, namely in the field of drug delivery (Sandolo et al., 2007). Natural antioxidants contained in the seed coat and pulp fruit are potential new products to be used in the food industry (Batista et al., 1996; Makris and Kefalas, 2004). Carob pods are actually explored as material for the production of bioethanol (Vourdoubas et al., 2002).

Carob has a significant economic importance in Algarve (Portugal), where are located some industrial companies of primary and secondary transformation, accounting for $14 \%$ $(40,100 \mathrm{t})$ of the world annual production (Graça and Custódio, 2000) with tendency to increase, due to the plantation of a high number of trees in the last 10 years.

Besides its economical importance this species has an increasing ecological significance as it can be used in reforestation of arid and degraded areas, growing well in marginal soils not adapted to other agricultural species (Winer, 1980). Owing to the low orchard management requirements the carob tree is suitable for part-time farming (Battle and Tous, 1997), contributing to the protection of the environment with low input demands, and low cost production (AAVV, 2002).

Several morphological studies have been published in the principal Mediterranean productive areas to assess the variation of several traits on the evaluation and characterization of carob cultivars (Coit, 1967; Orphanos and Papaconstantinou, 1969; Casanova et al., 1987; Crescimano et al., 1988; Battle and Tous, 1990; Albanell et al., 1996; Russo and Polignano, 1996) and correlation studies between morphological fruit and seed characters (Albanell et al., 1988, 1996; Caja et al., 1988). The principal carob cultivars from Portugal, namely 'Mulata', 'Galhosa', 'Canela', 'Spargale', 'Aida' and 'Lagoinha' were recently characterized based on 61 descriptors of the tree, leaf, inflorescence, fruit and seed (Barracosa and Graça, 2006).

The objectives of the present work were to characterize carob cultivars from the Algarve region using specifically fruit and seed phenotypic characters. Simultaneously, understand how fruit characteristics correlate with their own seeds in each cultivar, in order that a genetic improvement based on certain cultivars can be proposed aiming at the increase of their agroindustrial rentability.

\section{Materials and methods}

\subsection{Plant material}

The analysis was carried out on 15 carob trees belonging to the experimental field of the Regional Agricultural Centre of Algarve (DRAALG) located in Tavira (geographic locationlatitude: $37^{\circ} 06^{\prime} 58^{\prime \prime} \mathrm{N}$; longitude: $07^{\circ} 39^{\prime} 29^{\prime \prime} \mathrm{W}$ ), and included the principal Portuguese cultivars: 'Mulata'(accession no. 13), 'Costela Canela' (106), 'Costela Vaca' (107), 'Galhosa' (115), 'Brava Lagoa' (121), 'Lagoinha' (126), 'Canela' (129), 'Spargale' (134), 'Costa Loulé' (137), 'Gasparinha' (142),
'Pé Curto' (147), 'Alfarroba Burro' (149), 'Aida' (170), and in addition two Spanish cultivars 'Negra' (118), 'Rojal' (124).

\subsection{Fruit morphological analysis}

Thirteen fruit and seed continuous characters from 15 cultivars were pooled along the sampling years (1997-2003). The selection of the fruit pod and seed continuous characters for cultivar characterization (Table 2) was done by adapting the International Plant Genetic Resources Institute (IPGRI) descriptors (Battle and Tous, 1997). Annually, 25 pods were randomly collected from each tree and evaluated at peak maturity. Each fruit was manually decorticated and individually analysed. In each fruit pod the three heaviest seeds were selected. Seed characteristics, namely length, width and thickness, were ascertained individually for these seeds from each fruit, with the purpose of correlating fruit external morphological characteristics with seed characteristics.

\subsection{Statistical analysis}

One-way ANOVA was conducted for all the studied variables after testing for normality and homogeneity of variance. Differences between cultivar means were tested with Duncan's post hoc tests.

In order to find the main variation trends between fruit and seed characters in the carob cultivars and to evaluate their correlation, data were processed according to principal component analysis (PCA) using SPSS for Windows (Version 12.0, SPSS Inc., Chicago, IL, USA). Since the aim of PCA is to reveal common principles in the data, we pooled all sampling years (1997-2003) and used cultivar means in the multivariate analysis procedures. The PCA solution was accepted when eigenvalues where greater than one (Kaiser's criterion), and it was also compatible with the Cattell's scree rule. Component scores and factor loadings were calculated after variance maximizing axes rotation (Varimax rotation) and normalization. Only factor loadings equal or greater than 0.7 were considered as defining part of a principal component. Hierarchical cluster analysis (HCA) was utilized to investigate the similarities and dissimilarities among the cultivars with respect to seed and fruit characters. For classification, the Ward's Minimum Variance Method was utilized. The squared Euclidean distance was used as the dissimilarity measure for Ward's method. The grouping derived from HCA was used to interpret the results of the PCA score plots.

\section{Results}

\subsection{Morphological analysis}

The mean values for all the principal fruit and seed morphological characteristics observed were statistically different at a 1\%o level (Table 1), which indicates the high genetic diversity in the sampled cultivars. It can be seen from results of post hoc tests that fruit characters [width (FW), thickness (FT) and weight (FWe)] had the highest values in 
Table 1

Means, standard deviations (in brackets), " $F$ " values from one-way ANOVA and results of Duncan's multiple range test at the $5 \%$ level of 12 fruit and seed characters observed in 15 cultivars of $C$. siliqua Character Cultivars

\begin{tabular}{|c|c|c|c|c|c|c|c|c|c|c|c|c|c|c|c|c|}
\hline & 13 & 106 & 107 & 115 & 118 & 121 & 124 & 126 & 129 & 134 & 137 & 142 & 147 & 149 & 170 & \\
\hline $\mathrm{FL}$ & $\begin{array}{l}151.237 \mathrm{~h} \\
(11.470)\end{array}$ & $\begin{array}{l}156.970 \mathrm{~g} \\
(24.230)\end{array}$ & $\begin{array}{l}178.188 \mathrm{~d} \\
(15.526)\end{array}$ & $\begin{array}{l}151.650 \mathrm{~h} \\
(16.871)\end{array}$ & $\begin{array}{l}164.610 \mathrm{f} \\
(18.875)\end{array}$ & $\begin{array}{l}172.330 \mathrm{e} \\
(15.661)\end{array}$ & $\begin{array}{l}193.750 \mathrm{~b} \\
(21.281)\end{array}$ & $\begin{array}{l}201.700 \mathrm{a} \\
(25.136)\end{array}$ & $\begin{array}{l}129.500 \mathrm{I} \\
(13.289)\end{array}$ & $\begin{array}{l}159.677 \mathrm{~g} \\
(13.296)\end{array}$ & $\begin{array}{l}187.970 \mathrm{c} \\
(19.661)\end{array}$ & $\begin{array}{l}203.567 \mathrm{a} \\
(28.803)\end{array}$ & $\begin{array}{l}160.425 \mathrm{fg} \\
(13.542)\end{array}$ & $\begin{array}{l}161.557 \mathrm{fg} \\
(15.554)\end{array}$ & $\begin{array}{l}188.174 \mathrm{c} \\
(17.567)\end{array}$ & $86.653^{* * * *}$ \\
\hline FW & $\begin{array}{l}21.698 \mathrm{~cd} \\
(1.682)\end{array}$ & $\begin{array}{l}21.275 \mathrm{~d} \\
(1.843)\end{array}$ & $\begin{array}{l}19.888 \mathrm{~g} \\
(1.399)\end{array}$ & $\begin{array}{l}19.399 \mathrm{~b} \\
(1.977)\end{array}$ & $\begin{array}{l}22.645 \mathrm{~b} \\
(2.023)\end{array}$ & $\begin{array}{l}21.309 \mathrm{~d} \\
(1.620)\end{array}$ & $\begin{array}{l}19.998 \mathrm{fg} \\
(1.583)\end{array}$ & $\begin{array}{l}22.025 \mathrm{c} \\
(1.906)\end{array}$ & $\begin{array}{l}19.900 \mathrm{~g} \\
(1.152)\end{array}$ & $\begin{array}{l}20.331 \mathrm{f} \\
(1.584)\end{array}$ & $\begin{array}{l}23.380 \mathrm{a} \\
(1.416)\end{array}$ & $\begin{array}{l}19.137 \mathrm{~h} \\
(2.206)\end{array}$ & $\begin{array}{l}19.915 \mathrm{~g} \\
(1.571)\end{array}$ & $\begin{array}{l}16.161 \mathrm{I} \\
(1.769)\end{array}$ & $\begin{array}{l}20.725 \mathrm{e} \\
(1.766)\end{array}$ & *** \\
\hline FT & $\begin{array}{l}9.207 \mathrm{de} \\
(1.069)\end{array}$ & $\begin{array}{l}8.950 \mathrm{e} \\
(1.154)\end{array}$ & $\begin{array}{l}7.975 \mathrm{~h} \\
(1.342)\end{array}$ & $\begin{array}{l}10.860 \mathrm{a} \\
(1.564)\end{array}$ & $\begin{array}{l}10.049 \mathrm{~b} \\
(1.024)\end{array}$ & $\begin{array}{l}8.516 \mathrm{f} \\
(1.076)\end{array}$ & $\begin{array}{l}9.596 \mathrm{c} \\
(.996)\end{array}$ & $\begin{array}{l}10.003 \mathrm{~b} \\
(1.176)\end{array}$ & $\begin{array}{l}8.322 \mathrm{fg} \\
(.867)\end{array}$ & $\begin{array}{l}9.398 \mathrm{~cd} \\
(.940)\end{array}$ & $\begin{array}{l}10.987 \mathrm{a} \\
(1.463)\end{array}$ & $\begin{array}{l}7.191 \mathrm{I} \\
(1.343)\end{array}$ & $\begin{array}{l}8.320 \mathrm{f} \\
(1.253)\end{array}$ & $\begin{array}{l}7.304 \mathrm{I} \\
(.862)\end{array}$ & $\begin{array}{l}8.092 \mathrm{gh} \\
(1.074)\end{array}$ & **** \\
\hline FWe & $\begin{array}{l}17.468 \mathrm{de} \\
(2.582)\end{array}$ & $\begin{array}{l}17.122 \mathrm{e} \\
(3.540)\end{array}$ & $\begin{array}{l}19.885 \mathrm{c} \\
(3.246)\end{array}$ & $\begin{array}{l}17.347 \mathrm{de} \\
(3.808)\end{array}$ & $\begin{array}{l}19.737 \mathrm{c} \\
(3.523)\end{array}$ & $\begin{array}{l}19.313 \mathrm{c} \\
(3.505)\end{array}$ & $\begin{array}{l}19.974 \mathrm{c} \\
(4.750)\end{array}$ & $\begin{array}{l}23.707 \mathrm{~b} \\
(4.790)\end{array}$ & $\begin{array}{l}11.463 \mathrm{~g} \\
(1.252)\end{array}$ & $\begin{array}{l}19.108 \mathrm{c} \\
(3.127)\end{array}$ & $\begin{array}{c}26.395 \mathrm{a} \\
(4.885)\end{array}$ & $\begin{array}{l}19.617 \mathrm{c} \\
(4.548)\end{array}$ & $\begin{array}{l}17.459 \text { de } \\
(2.220)\end{array}$ & $\begin{array}{l}13.320 \mathrm{f} \\
(2.234)\end{array}$ & $\begin{array}{l}18.218 \mathrm{~d} \\
(4.080)\end{array}$ & $137.388^{* * * *}$ \\
\hline NS & $\begin{array}{l}10.148 \mathrm{~h} \\
(1.605)\end{array}$ & $\begin{array}{r}10.31 \mathrm{~h} \\
(2.377)\end{array}$ & $\begin{array}{l}13.656 \mathrm{a} \\
(1.667)\end{array}$ & $\begin{array}{l}11.371 \mathrm{f} \\
(2.277)\end{array}$ & $\begin{array}{l}11.160 \mathrm{fg} \\
(1.881)\end{array}$ & $\begin{array}{l}12.510 \mathrm{de} \\
(2.077)\end{array}$ & $\begin{array}{l}12.257 \mathrm{de} \\
(2.731)\end{array}$ & $\begin{array}{l}12.475 \text { de } \\
(2.240)\end{array}$ & $\begin{array}{l}12.178 \mathrm{e} \\
(1.320)\end{array}$ & $\begin{array}{l}13.452 \mathrm{ab} \\
(2.353)\end{array}$ & $\begin{array}{l}13.690 \mathrm{a} \\
(2.202)\end{array}$ & $\begin{array}{l}13.096 \mathrm{bc} \\
(2.059)\end{array}$ & $\begin{array}{l}12.569 \mathrm{de} \\
(2.423)\end{array}$ & $\begin{array}{l}10.821 \mathrm{~g} \\
(2.150)\end{array}$ & $\begin{array}{l}12.910 \mathrm{~cd} \\
(1.828)\end{array}$ & $47.151^{* * *}$ \\
\hline NA & $\begin{array}{l}2.185 \mathrm{c} \\
(1.746)\end{array}$ & $\begin{array}{l}2.570 \mathrm{~b} \\
(1.736)\end{array}$ & $\begin{array}{l}1.494 \mathrm{f} \\
(1.116)\end{array}$ & $\begin{array}{l}1.925 \mathrm{~cd} \\
(1.460)\end{array}$ & $\begin{array}{l}2.170 \mathrm{c} \\
(1.258)\end{array}$ & $\begin{array}{l}1.310 \mathrm{f} \\
(1.517)\end{array}$ & $\begin{array}{l}3.216 \mathrm{ab} \\
(2.508)\end{array}$ & $\begin{array}{l}2.838 \mathrm{ab} \\
(1.733)\end{array}$ & $\begin{array}{l}1.326 \mathrm{f} \\
(1.270)\end{array}$ & $\begin{array}{l}1.948 \mathrm{~cd} \\
(1.712)\end{array}$ & $\begin{array}{l}2.130 \mathrm{c} \\
(1.539)\end{array}$ & $\begin{array}{l}1.795 \text { cde } \\
(1.238)\end{array}$ & $\begin{array}{l}1.320 \mathrm{de} \\
(1.310)\end{array}$ & $\begin{array}{l}3.029 \mathrm{~cd} \\
(1.795)\end{array}$ & $\begin{array}{l}1.677 \mathrm{def} \\
(1.110)\end{array}$ & $20.596^{* * * *}$ \\
\hline $\mathrm{Y}$ & $\begin{array}{l}12.420 \mathrm{fg} \\
(2.433)\end{array}$ & $\begin{array}{l}10.955 \mathrm{ij} \\
(2.665)\end{array}$ & $\begin{array}{l}12.412 \mathrm{fg} \\
(2.137)\end{array}$ & $\begin{array}{l}13.738 \text { de } \\
(2.946)\end{array}$ & $\begin{array}{l}12.539 \mathrm{fg} \\
(1.747)\end{array}$ & $\begin{array}{l}13.558 \mathrm{e} \\
(2.664)\end{array}$ & $\begin{array}{l}10.891 \mathrm{ij} \\
(3.718)\end{array}$ & $\begin{array}{l}10.849 \mathrm{j} \\
(2.871)\end{array}$ & $\begin{array}{l}18.141 \mathrm{a} \\
(2.128)\end{array}$ & $\begin{array}{l}12.735 \mathrm{f} \\
(2.618)\end{array}$ & $\begin{array}{l}11.517 \mathrm{hi} \\
(2.121)\end{array}$ & $\begin{array}{l}11.875 \mathrm{gh} \\
(2.788)\end{array}$ & $\begin{array}{l}14.732 \mathrm{c} \\
(2.536)\end{array}$ & $\begin{array}{l}16.566 \mathrm{~b} \\
(3.068)\end{array}$ & $\begin{array}{l}13.791 \mathrm{~cd} \\
(2.965)\end{array}$ & t \\
\hline SL & $\begin{array}{l}9.624 \mathrm{de} \\
(.573)\end{array}$ & $\begin{array}{l}9.540 \mathrm{e} \\
(.436)\end{array}$ & $\begin{array}{l}9.948 \mathrm{~b} \\
(.514)\end{array}$ & $\begin{array}{l}9.328 \mathrm{fg} \\
(.351)\end{array}$ & $\begin{array}{l}9.790 \mathrm{c} \\
(.453)\end{array}$ & $\begin{array}{c}10.207 \mathrm{a} \\
(.329)\end{array}$ & $\begin{array}{l}9.906 \mathrm{~b} \\
(.418)\end{array}$ & $\begin{array}{l}9.350 \mathrm{f} \\
(.416)\end{array}$ & $\begin{array}{l}9.787 \mathrm{c} \\
(.417)\end{array}$ & $\begin{array}{l}9.195 \mathrm{gh} \\
(.490)\end{array}$ & $\begin{array}{c}10.155 \mathrm{a} \\
(.320)\end{array}$ & $\begin{array}{l}9.716 \mathrm{~cd} \\
(.545)\end{array}$ & $\begin{array}{l}9.636 \mathrm{de} \\
(.328)\end{array}$ & $\begin{array}{l}9.025 \mathrm{~h} \\
(.434)\end{array}$ & $\begin{array}{l}8.660 \mathrm{I} \\
(.369)\end{array}$ & $139.749^{* * * *}$ \\
\hline SW & $\begin{array}{l}7.474 \mathrm{~b} \\
(.323)\end{array}$ & $\begin{array}{l}6.822 \mathrm{~g} \\
(.498)\end{array}$ & $\begin{array}{l}7.001 \mathrm{f} \\
.539)\end{array}$ & $\begin{array}{l}7.391 \mathrm{c} \\
(.343)\end{array}$ & $\begin{array}{l}7.663 \mathrm{a} \\
(.475)\end{array}$ & $\begin{array}{l}7.131 \mathrm{e} \\
(.279)\end{array}$ & $\begin{array}{l}6.960 \mathrm{f} \\
.321)\end{array}$ & $\begin{array}{l}7.514 \mathrm{~b} \\
(.394)\end{array}$ & $\begin{array}{l}6.983 \mathrm{f} \\
(.341)\end{array}$ & $\begin{array}{l}6.688 \mathrm{~h} \\
(.369)\end{array}$ & $\begin{array}{l}7.498 \mathrm{~b} \\
(.266)\end{array}$ & $\begin{array}{l}6.373 \mathrm{i} \\
.(344)\end{array}$ & $\begin{array}{l}7.284 \mathrm{~cd} \\
(.366)\end{array}$ & $\begin{array}{l}7.285 \mathrm{c} \\
(.341)\end{array}$ & $\begin{array}{l}7.241 \mathrm{de} \\
(.324)\end{array}$ & $9^{* * * *}$ \\
\hline ST & $\begin{array}{l}4.589 \text { ef } \\
(1.155)\end{array}$ & $\begin{array}{l}3.901 \mathrm{j} \\
(0.400)\end{array}$ & $\begin{array}{l}3.949 \mathrm{ij} \\
(.231)\end{array}$ & $\begin{array}{l}5.181 \mathrm{a} \\
(.937)\end{array}$ & $\begin{array}{l}4.166 \text { de } \\
(.222)\end{array}$ & $\begin{array}{l}4.061 \mathrm{gh} \\
(.230)\end{array}$ & $\begin{array}{l}3.7601 \\
(.275)\end{array}$ & $\begin{array}{l}4.226 \mathrm{~d} \\
(.314)\end{array}$ & $\begin{array}{l}4.079 \mathrm{fg} \\
(.338)\end{array}$ & $\begin{array}{l}4.003 \mathrm{hi} \\
(.252)\end{array}$ & $\begin{array}{l}4.174 \mathrm{de} \\
(.183)\end{array}$ & $\begin{array}{l}4.039 \mathrm{gh} \\
(.330)\end{array}$ & $\begin{array}{l}4.337 \mathrm{c} \\
(.309)\end{array}$ & $\begin{array}{l}4.401 \mathrm{~b} \\
(.319)\end{array}$ & $\begin{array}{l}4.373 \mathrm{de} \\
(.513)\end{array}$ & $114.733^{\text {**** }}$ \\
\hline TSWe & $\begin{array}{l}2.148 \mathrm{~d} \\
(.416)\end{array}$ & $\begin{array}{l}1.838 \mathrm{f} \\
(.506)\end{array}$ & $\begin{array}{l}2.431 \mathrm{c} \\
.393)\end{array}$ & $\begin{array}{l}2.345 \mathrm{c} \\
(.545)\end{array}$ & $\begin{array}{l}2.459 \mathrm{bc} \\
(.484)\end{array}$ & $\begin{array}{l}2.568 \mathrm{~b} \\
(.441)\end{array}$ & $\begin{array}{l}2.157 \mathrm{~d} \\
(.510)\end{array}$ & $\begin{array}{l}2.510 \mathrm{~b} \\
(.616)\end{array}$ & $\begin{array}{l}2.018 \mathrm{e} \\
(.337)\end{array}$ & $\begin{array}{l}2.413 \mathrm{c} \\
(.520)\end{array}$ & $\begin{array}{l}2.986 \mathrm{a} \\
(.490)\end{array}$ & $\begin{array}{l}2.229 \mathrm{~d} \\
(.353)\end{array}$ & $\begin{array}{l}2.550 \mathrm{~b} \\
(.436)\end{array}$ & $\begin{array}{l}2.159 \mathrm{~d} \\
(.471)\end{array}$ & $\begin{array}{l}2.550 \mathrm{~b} \\
(.444)\end{array}$ & $51.685^{* * *}$ \\
\hline ISWe & $\begin{array}{l}.211 \mathrm{~b} \\
(.021)\end{array}$ & $\begin{array}{l}.173 \mathrm{~h} \\
(.033)\end{array}$ & $\begin{array}{l}.179 \mathrm{gh} \\
(.018)\end{array}$ & $\begin{array}{l}.208 \mathrm{bc} \\
(.021)\end{array}$ & $\begin{array}{l}.219 \mathrm{a} \\
(.023)\end{array}$ & $\begin{array}{l}.205 \mathrm{~cd} \\
(.018)\end{array}$ & $\begin{array}{l}.175 \mathrm{gh} \\
(.019)\end{array}$ & $\begin{array}{l}.200 \mathrm{de} \\
(.033)\end{array}$ & $\begin{array}{l}.166 \mathrm{I} \\
(.021)\end{array}$ & $\begin{array}{l}.179 \mathrm{gh} \\
(.022)\end{array}$ & $\begin{array}{l}.218 \mathrm{a} \\
(.013)\end{array}$ & $\begin{array}{l}.166 \mathrm{i} \\
(.017)\end{array}$ & $\begin{array}{l}.198 \text { ef } \\
(.015)\end{array}$ & $\begin{array}{l}.200 \mathrm{def} \\
(.021)\end{array}$ & $\begin{array}{l}.198 \mathrm{f} \\
(.024)\end{array}$ & $112.673^{* * * *}$ \\
\hline
\end{tabular}

Statistically significant differences between cultivars at $p \leq 0.001$. 
Table 2

Factor loadings for each variable on the components of PCA analysis

\begin{tabular}{lccc}
\hline Variable & PC1 & PC2 & PC3 \\
\hline Fruit length, FL (mm) & -.56 & .51 & .12 \\
Fruit width, FW (mm) & .78 & .302 & .03 \\
Fruit thickness, FT (mm) & .70 & -.36 & .20 \\
Fruit weight, FWe (g) & .95 & .25 & .04 \\
Number of seeds, NS & .35 & .58 & -.62 \\
Number of aborted seeds, NA & .10 & -.14 &. $\mathbf{8 5}$ \\
Yield, Y (\%) & -.67 & -.41 & -.55 \\
Seed length, SL (mm) & .34 & .32 & -.11 \\
Seed width, SW (mm) & .46 & -.81 & .03 \\
Seed thickness, ST (mm) & -.03 & -.80 & -.25 \\
Total seed weight, TSWe (g) & .77 & -.11 & -.55 \\
Individual seed weight, ISWe (g) & .59 & -.74 & -.08 \\
Eigen values & 4.156 & 2.930 & 1.853 \\
\% of variance & 34.6 & 24.4 & 15.4 \\
Cumulative \% & 34.6 & 59.0 & 74.5 \\
\hline
\end{tabular}

Factor loadings $>0.7$ are in bold.

'Lagoinha' (126) and 'Costa Loulé' (137) cultivars and the lowest values in 'Canela' (129) and 'Alfarroba Burro' (149). Seed characters like seed width (SW) and individual seed weight (ISWe) exhibit the highest values in cultivars 'Mulata' (13), 'Negra' (118) and 'Costa Loule' (137) and the lowest in 'Costela Canela' (106), 'Spargale' (134) and 'Gasparinha' (142) cultivars. Finally, the number of abortive seeds (NA) was significantly higher in cultivars 'Rojal' (124), 'Lagoinha' (126) and 'Alfarroba Burro' (149) and lower in 'Costela Vaca' (107), 'Brava Lagoa' (121) and 'Canela' (129).

An acceptable solution for principal component analysis was reached when three dimensions of the model were found to be significant and explained $74.5 \%$ of the total variance of the original variables set (Table 2). The first component (PC1), accounting for $34.6 \%$ of the total variance, is dominated by fruit characters, namely width (FW), thickness (FT) and weight (FWe). In the second component (PC2), seed characters, width (SW), thickness (ST) and individual seed weight (ISWe), characterised by a high negative loading were the main contributors and explained $24.4 \%$ of total variance. Finally, the

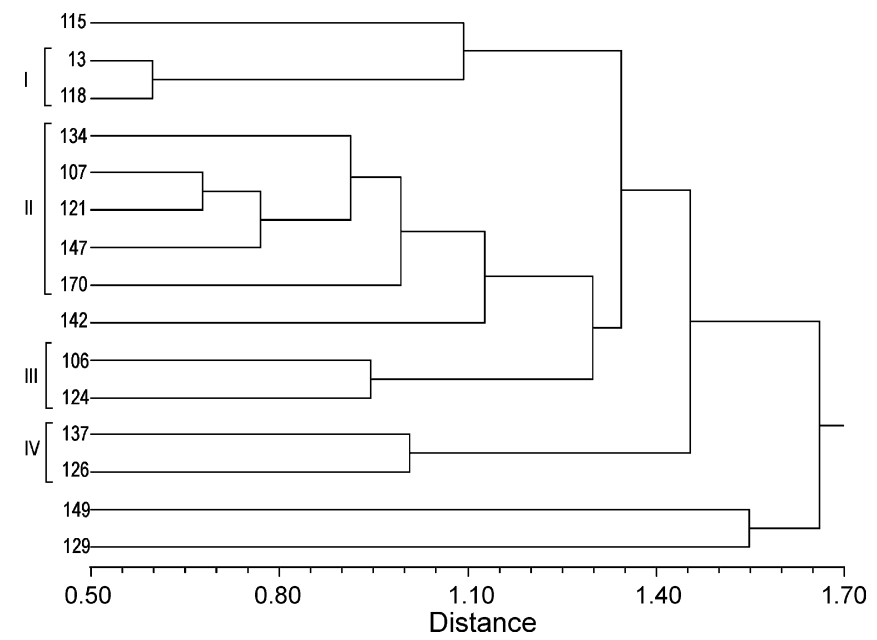

Fig. 1. Dendrogram for the classification of 15 cultivars of $C$. siliqua according to the 13 fruit and seed characters from PCA.

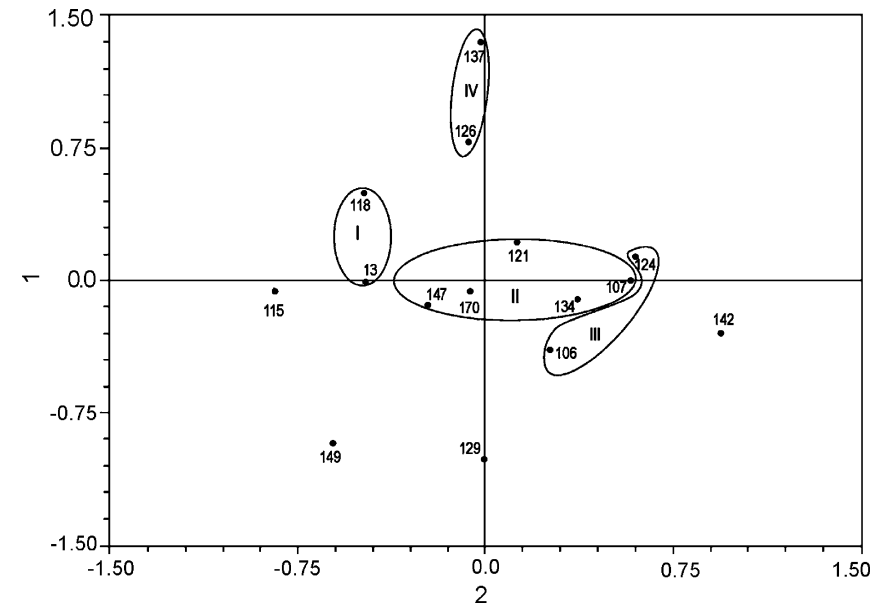

Fig. 2. Projection of score of 15 carob cultivars onto the plane defined by the principal coordinates (1-2).

third principal component (PC3) is dominated by the number of abortive seeds (NA).

By applying cluster analysis to variables retained by PCA dendogram in Fig. 1, four groups of cultivars were differentiated at a dissimilarity level of 1.0. Cluster I consisted of 'Mulata' (13) and 'Negra' (118) cultivars. 'Costela Vaca' (107), 'Brava Lagoa' (121), 'Spargale' (134), 'Pé Curto' (147) and 'Aida' (170) were placed in cluster II; Cluster III included 'Costela Canela' (106) and 'Rojal' (124); 'Costa Loulé' (137) and 'Lagoinha' (126) were placed in cluster IV. Cultivars 'Galhosa' (115), 'Canela' (129), 'Gasparinha' (142) and 'Alfarroba Burro' (149) can be considered as rather singular.

Principal component and cluster analysis discriminated the sampled cultivars in four clusters (Fig. 2) using the first two principal components and accounted for about $59 \%$ of the total variability among the carob cultivars, based on fruit and seed traits, respectively.

Cluster I consisted of cultivars 'Mulata' (13) and 'Negra' (118). Cluster II including 'Costela Vaca' (107), 'Brava Lagoa' (121), 'Spargale' (134), 'Pé Curto' (147) and 'Aida' (170)

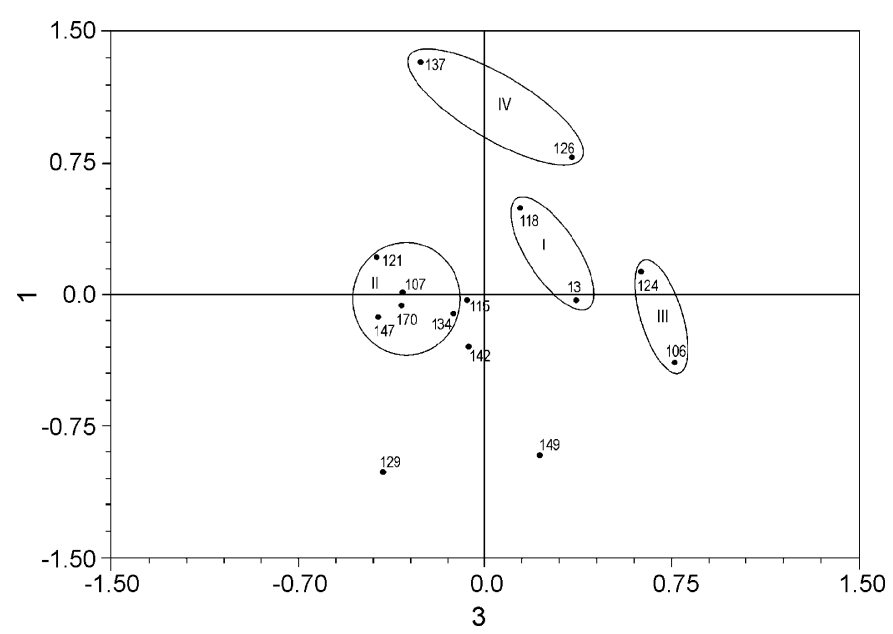

Fig. 3. Projection of score of 15 carob cultivars onto the plane defined by the principal coordinates (1-3). 
cultivars are plotted on a central position (Fig. 2). The cultivars 'Costela Canela' (106) and 'Rojal' (124) of cluster III appear clearly separated from cluster II using the principal components 1 and 3 (Fig. 3), determined by the high number of aborted seeds of these cultivars. 'Lagoinha' (126) and 'Costa Loulé' (137) cultivars of cluster IV are placed on the upper left quadrant.

'Galhosa' (115), 'Canela' (129) and 'Alfarroba Burro' (149) cultivars were plotted ungrouped on the left-lower quadrant and 'Gasparinha' (142) cultivar is plotted on the right-lower quadrant (Fig. 2).

\section{Correlation analysis}

From the morphological study, significant Pearson correlation coefficients $(P<0.05-0.001)$ were obtained by cultivar, between the more significant morphological characteristics from pod and its seeds (FL, FW, FWe, Y, NS, SL, SW, ST, ISWe and TSWe) used for cultivars comparison, as shown in Table 3. These correlations are important for the evaluation of agroindustrial rentability.

The majority of the cultivars showed non-significant correlation between fruit length and seed yield (FL-Y). However, the cultivars ['Gasparinha' (142) and 'Canela' (129)] showed a significant negative correlation. All cultivars except 'Gasparinha' (142) had significant correlation between fruit length and number of seeds (FL-NS), which ranged (between 0.23 and 0.76). 'Gasparinha' (142) cultivar showed the highest positive correlation coefficients $(0.5)$ between fruit length and two of seed morphological characteristics, length and width.

No cultivars presented positive correlations between fruit length and seed thickness (FL-ST). 'Lagoinha' (126) cultivar showed the highest negative correlation $(-0.5)$.

The cultivars 'Lagoinha' (126), 'Canela' (129) and 'Alfarroba Burro' (149) presented a negative correlation between fruit length and individual seed weight (FL-ISWe) and consequently exhibited non-significant correlations between fruit length and total seed weight (FL-TSWe). The highest correlation coefficients (0.7) observed between (FLTSWe) were in 'Galhosa' (115), 'Negra' (118), 'Costa Vaca' (137) and 'Aida' (170) cultivars and are directly related with the high correlations $(0.7)$ obtained in these cultivars between (FLNS).

Cultivars 'Negra' (118), 'Rojal' (124), 'Lagoinha' (126) and 'Aida' (170), presented simultaneously high correlation coefficients between fruit width and seed width (FW-SW) and between fruit width and individual seed weight (FWISWe). Similarly, cultivars 'Aida' (170), 'Negra' (118) and 'Costela Canela' (106) presented the highest correlation coefficients between fruit weight and seed width (FWe-W) and between fruit weight and individual seed weight (FWeISWe).

All cultivars showed a negative correlation between fruit weight and seed yield (FWe-Y) and the cultivars with the heaviest fruits presented the highest negative correlation coefficients.

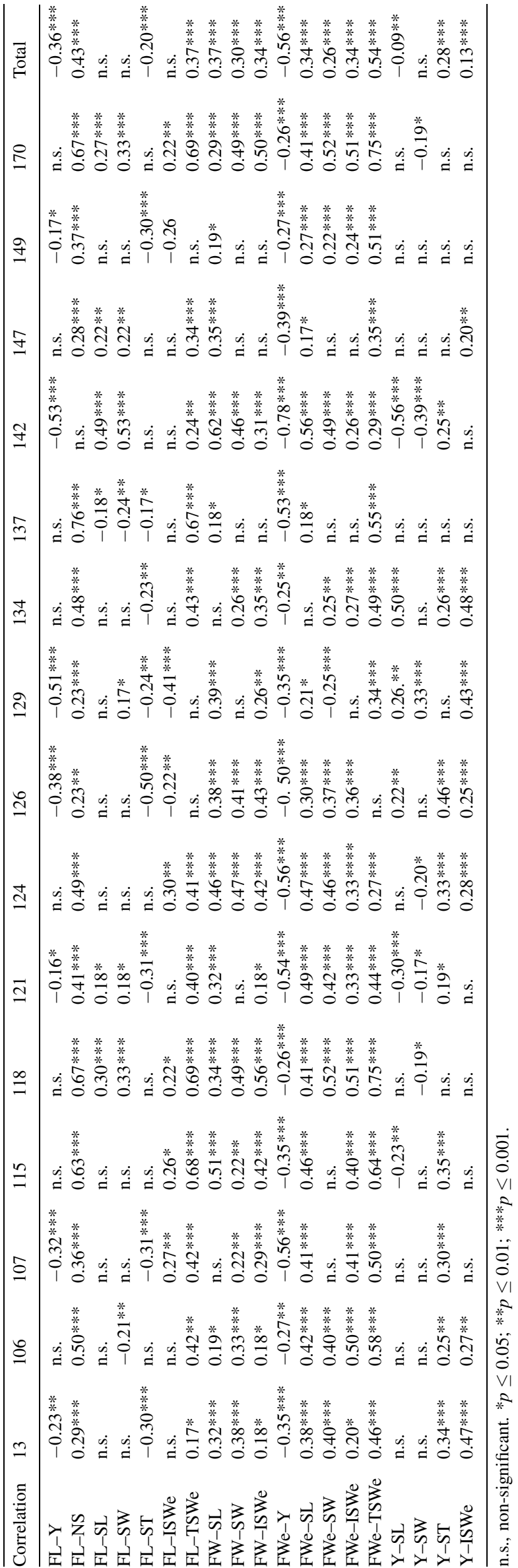


All cultivars but 'Lagoinha' (126), showed significant low to high correlation coefficients $(0.27-0.75)$ between fruit weight and total seed weight (FWe-TSWe). Cultivars 'Negra' (118), 'Aida' (170), 'Galhosa' (115) and 'Costela Canela' (106) presented the highest values.

In the present work, 'Lagoinha' (126) cultivar showed the highest correlation coefficient $(0.46)$ between seed thickness and fruit seed yield (Y-ST).

The highest correlation coefficient (0.5) between $\mathrm{Y}$ and ISWe was observed for 'Spargale' (134) and 'Mulata' (13) cultivars. The individual seed weight seems fundamental to increase seed yield in these two cultivars. The large majority of the cultivars presented non-significant coefficient.

\section{Discussion}

Carob cultivars can be characterised using a combination of phenotypical and genotypical characteristics. Morphological and physiological characters are important and have been traditionally used for the identification of carob varieties (Tous and Battle, 1990). However, traditional cultivar identification based on morphological and physiological traits, requires a large set of phenotypical data that are often difficult to assess and frequently prone to large environmental induced variation. To avoid unreliable determinations, the presented data were obtained over 7 years (1997-2003) from trees belonging to a field carob collection. These cultivars had a large geographic provenance over the Algarve region, adapted to different microclimatic conditions.

The values of morphological traits obtained by cultivar in the present study were compared with those from other countries of the Mediterranean basin namely, Cyprus Orphanos and Papaconstantinou, 1969, Spain (Sanchez-Capuchino et al., 1988; Tous and Battle, 1990), Greece (Marakis et al., 1988) and Italy (Donno and Pannaro, 1965; Russo and Polignano, 1996). 'Negra' cultivar from Spain (Tous and Battle, 1990) presented high similarity in morphological characteristics of the fruit with 'Negra' (118) and with 'Mulata' (13) cultivars from the present study. As 'Mulata' cultivar from the Algarve region, 'Negra' cultivar in Valencia region showed a high level of fruit morphological polymorphism (Sanchez-Capuchino et al., 1988). As a result of the human influence, these two cultivars represent the large majority of the grafted trees existent in the Algarve (Portugal) and Catalonia (Spain) regions, respectively. The cultivar 'Rojal' analysed by Tous and Battle (1990) showed a high homology of the external fruit morphology with those of the present study. However, seeds revealed a very higher individual weight $(0.260 \mathrm{~g})$ than that found in the present survey $(0.175 \mathrm{~g})$.

'Galhosa', the second most representative cultivar in Algarve region, better adapted for higher altitude and arid conditions, presented a high similarity of fruit and seed characteristics with the 'Koundourka' variety from Cyprus described by Orphanos and Papaconstantinou (1969) and with the 'Sretta' cultivar from the Bari region (Italy) (Donno and Pannaro, 1965).

Principal component and cluster analysis discriminated the sampled cultivars in four clusters. Cluster I is characterized by large fruits with medium length, weight and seed yield. The seeds, in reduced number per fruit, are individually very large and heavy in both cultivars. Cultivars of cluster II presented fruits with a high total seed weight and seed yield. Marked differences in fruit length were evident among the cultivars of this cluster. The seeds were characterised by a high similarity in total weight per fruit. However they presented a different shape, elongated in 'Costela Vaca' (107) and 'Brava Lagoa' (121) and round in 'Aida' (170). Trees of cluster III showed a high number of aborted seeds and a medium seed yield per fruit as result of low individual seed weight as a result of long narrow shape with low thickness. Cultivars of cluster IV showed very long, large and heavy fruits. The seeds were also very large and heavy. The high number of seeds resulted in a very high total seed weight and a medium seed yield per fruit. The ungrouped cultivars 'Galhosa' (115), 'Canela' (129) and 'Alfarroba Burro' (149) are characterized by fruits with high seed yield and the seeds of 'Galhosa' (115) and 'Alfarroba Burro' (149) cultivars were round shaped with a medium individual weight. 'Gasparinha' (142) cultivar presented very long fruits with high number of seeds of low individual weight.

Data obtained from morphological studies have been used to provide total correlation coefficients between morphological fruit and seed characters (Albanell et al., 1988, 1996; Caja et al., 1988). The total correlation in the present study showed similar coefficients or a general identical tendency concerning the principal parameters. However, a wide range of values were observed for each correlation when cultivars were considered separately (Table 3 ). The correlation analyses established by cultivar provided a specific understanding about the way how fruit and seed characteristics correlates within each cultivar. This approach can be useful for the development of a breeding programme aiming at the selection of cultivars in order to increase the seed yield, seed thickness, individual and total seed weight by fruit, characteristics that are determinant to improve the industrial exploitation. This must obviously be done in close agreement with agricultural practices.

In Spanish cultivars, Albanell et al. (1996) observed a high correlation coefficient between fruit length and total seed weight (0.60). In the present study several cultivars showed low to non-significant coefficients, namely the cultivars with the highest seed yield ['Canela' (129) and 'Alfarroba Burro' (149)]. However, Portuguese cultivars ['Galhosa' (115), 'Costa Vaca' (137), 'Aida' (170)] and 'Negra' (118) from Spain showed a high coefficient correlations (0.7).

Albanell et al. (1996) reported a high similarity in the number of seed by pod in Spanish and Cypriots carob trees, with a ratio of kernels/cm pod length of 0.61 and 0.63 , respectively. This indicates that the number of seeds should vary between fixed limits and is therefore a feature of this species (Albanell et al., 1996). In the present study the observed mean value was slightly higher (0.72); however, significant differences were obtained between cultivars: 'Canela' (129) exhibits the highest value (0.95) and 'Lagoinha' (126) the lowest (0.62).

There is no relation between the individual seed weight and the ratio of kernels/cm pod length. The cultivars with the lowest individual seed weight (0.166 g) 'Canela' (129) and 
'Gasparinha' (142) presented extreme ratio values with 0.95 and 0.64 , respectively.

Cultivars with non-significant correlation between fruit length and seed yield (FL-Y) presented a high number of seeds ['Costa Loulé' (137)], or heavy seeds ['Negra' (118)]. On the contrary, the cultivars with the lowest individual seed weight ['Gasparinha' (142) and 'Canela' (129)] showed a significant negative correlation, because the individual seeds weight seems not compensate the increment of fruit length. 'Galhosa' (115), 'Pé Curto' (147), 'Alfarroba Burro' (149) and 'Aida' (170) cultivars plotted on the left lower quadrant (Fig. 2) presented non-significant correlation coefficients between FL and Y meaning that their seed yield is not reduced by the fruit length.

The cultivar 'Gasparinha' (142) with the longest fruit and thinnest seeds had non-significant correlation between fruit length and number of seeds (FL-NS) and the cultivars 'Costa Loulé' (137) and 'Negra' (118) with largest and heaviest individual seeds had the highest correlation coefficients.

In cultivars 'Lagoinha' (126), 'Canela' (129) and 'Alfarroba Burro' (149) the increase of the fruit length seems to provoke a reduction in the individual seed weight.

In cultivars 'Negra' (118), 'Aida' (170), 'Galhosa' (115) and 'Costela Canela' (106) heavier fruits seem to promote heavier individual seeds, because high coefficients values were found for the correlations between fruit weight and individual seed weight (FWe-ISWe), and fruit weight and total seed weight (FWe-TSWe).

General correlation studies showed that seed thickness correlates positively with the seed yield of the fruit $(0.46)$ (Albanell et al., 1996). In the present work, 'Lagoinha' (126) cultivar showed the highest correlation coefficient (0.46). However, the cultivars of clusters II and III plotted on the upper and lower-right quadrants (Fig. 2) presented slightly lower values (0.3) and cultivars 'Canela' (129), 'Pé Curto' (147), 'Alfarroba Burro' (149), 'Aida' (170) plotted on lower left quadrant had non-significant correlation.

Albanell et al. (1996) showed that seed yield correlates negatively with fruit weight $(-0.33)$. In the present survey all cultivars had a negative correlation coefficient ranging from -0.25 to -0.78 and cultivars with the heaviest fruits presented the highest negative coefficients.

In Spanish cultivars, Albanell et al. (1996) observed a low correlation coefficient (0.19) between seed yield and individual seed weight (Y-ISWe). The present study showed a similar total correlation coefficient (0.13). However, the analysis carried out specifically by cultivar showed a large variance of the correlation coefficient (from non-significant to 0.48).

The cultivars plotted on the lower left quadrant (Fig. 2), namely 'Mulata' (13), 'Galhosa' (115), 'Pé Curto' (147), 'Alfarroba Burro' (149) and 'Aida' (170) are more appropriate for industrial gum exploitation due to the conjugation of their fruit and seed characteristics, with thick seeds and high seed yield. However, seeds from 'Canela' (129) cultivar that plotted on this quadrant, are of low interest for industry application because they are long shaped and have a rough episperm.

The present findings on the characterization of Portuguese carob cultivars that exhibited diverse patterns of fruit and seed characteristics could be the basis of a programme, preferentially assisted by a molecular genetic marker approach, aiming at the genetic improvement of this taxon, and at the optimization of its industrial exploitation.

Moreover, the development of varieties more adapted to actual and future demands of industry must take in consideration a strategy that prevents genetic erosion. The domestication that took place in Algarve has been based on 'Mulata' cultivar, thus the reduction of the biodiversity of this species in this region is a real risk. Thus, evaluation of the biodiversity in non-grafted carob trees dispersed all over the region is a fundamental step for the implementation of a conservation strategy.

\section{Acknowledgements}

This work was supported by an EEC grant PRAXIS XXI/ BD/3601/94 of Foundation for Science and Technology (FCT). We are very grateful to Brito Carvalho, José Graça and to Manuel Caetano from "Associação Interprofissional para o Desenvolvimento e Valorização da Alfarroba (AIDA).

\section{References}

AAVV, 2002. I frutttiferi minori in Europa. A cura di E. Billini. Ed. L'Informatore Agrario, Verona, p. 191.

Albanell, E., Caja, G., Casanova, R., 1988. Características físicas y contenido en endospermo de semillas españolas de algarrobo (Ceratonia siliqua L.). In: Proceedings of the II International carob symposium, Valencia, Spain, September 29-October 1, pp. 558-566.

Albanell, E., Caja, G., Plaixats, J., 1996. Characterization of carob fruits (Ceratonia siliqua L.), cultivated in Spain for Agroindustrial use. Int. Tree Crops J. 9, 1-9.

Barracosa, P., Graça, J., 2006. Fichas de caracterização-Alfarrobeira. In: Caracterização de variedades regionais Portuguesas. Ministério da Agricultura, do Desenvolvimento Rural e Pescas. Direcção-Geral de Protecção das Culturas. Oeiras, Portugal, pp. 1-16.

Batista, M.T., Amaral, M.T., Proença Da Cunha, A., 1996. Carob fruits as source of natural oxidants. In: Proceedings of the Communication in Third International Carob Symposium, Tavira, Portugal, June, pp. 19-23.

Battle, I., Tous, J., 1990. Cultivares autóctonos de algarrobo (Ceratonia siliqua L.) en Cataluña. Investigación Agrária 5 (2), 223-238.

Battle, I., Tous, J., 1997. Carob tree. Ceratonia siliqua L. Promoting the conservation and use of underutilized crops. Institut of plant genetics and crop plant research, Genetic Resources Institute, Rome, Italy.

Caja, G., Albanell, E., Casanova, R., 1988. Caracterización morfológica de frutos de algarrobo cultivados en España. In: Proceedings of the II International Carob symposium, Valencia, Spain, September 29-October 1, pp. 119-129.

Carlson, W.A., 1986. The carob: evaluation of trees, pods and kernels. Int. Tree Crops J. 3, 281-290.

Casanova, R., Albanel, E., Caja, G., 1987. Valoracion morfologica de los frutos de los frutos de variedades de algarrobo (Ceratonia siliqua $\mathrm{L}$.) cultivadas en España. In: Proceedings of the Abstracts of the II International carob symposium, Valencia, Spain, September 29-October 1, pp. A-5-A-9.

Coit, J.E., 1967. Carob varieties for the semi-arid southwest. Fruit Variet. Hort. Digest $21,5-9$

Crescimano, F.G., De Michele, A., Di Lorenzo, R., Occorso, G., Raimondo, A., 1988. Aspetti morfologici e carpologici di cultivar di carrubo (Ceratonia siliqua L.). In: Proceedings of the II International carob symposium, Valencia, Spain, September 29-October 1, pp. 169-181.

Donno, G., Pannaro, A.D., 1965. Su alcune cultivar di carrubo della provincial di Bari e proposta de una scheda descriptiva (Primo contributo). Scienza e Tecnica Agraria vol. V, N. 4-5:3-39. 
Graça, J., Custódio, S., 2000. Caracterização da Fileira da Alfarroba. In: Sistemas Agrários Tradicionais no Algarve. Contributos para o seu Estudo. Direcção Regional de Agricultura do Algarve. Faro, Portugal, pp. 99-157.

Hillcoat, D., Lewis, G., Verdcourt, B., 1980. A new species of Ceratonia (Leguminosae-Caesalpinioideae) from Arabia and Somali Republic. Kew Bull. 35 (2), 261-271.

Makris, D., Kefalas, P., 2004. Carob pods (Ceratonia siliqua L.) as a source of polyphenolic antioxidants. Food Technol. Biotechnol. 42 (2), 105-108.

Marakis, S., Kalaitzakis, J., Mitrakos, K., 1988. Criteria for recognizing carob tree varieties. In: Proceedings of the II. International Carob symposium, Valencia, Spain, pp. 195-208.

Mitrakos, K., 1987. La botanica del Ceratonia siliqua L. In: Proceedings of the II International Carob symposium, Valencia, Spain, September 29-October 1, pp. 209-218.

Orphanos, P.I., Papaconstantinou, J., 1969. The Carob Varieties of Cyprus. Tech. Bull., 5. Cyprus Agricultural Research Institute, Nicosia.

Russo, G., Polignano, G.B., 1996. Variation of seed and fruit characters in Ceratonia siliqua L. cultivars. Genet. Resourc. Crop Evol. 43, 525553.
Sanchez-Capuchino, J.A., Salazar, D., Garcia, S., Matinez, R., Melgarejo, P., 1988. Tipificacion pomológica de los algarrobos de la Comunidad Valenciana. In: Proceedings of the II International Carob symposium, Valencia, Spain, September 29-October 1, pp. 69-79.

Sandolo, C., Coviello, T., Matricardi, P., Alhaique, F., 2007. Caracterization of polysaccharide hydrogels for modified drug delivery. Eur. Biophys. J. 36 (7), 693-700.

Tous, J., Battle, I., 1990. El algarrobo. Ed Mundi-Prensa, Madrid, Spain.

Tucker, S.C., 1992. The development basis for sexual expression in Ceratonia siliqua L. (Leguminosae: Caesalpinioideae: Cassieae). Am. J. Bot. 79, 318327.

Vourdoubas, J., Makris, P., Kefalas, J., Kaliakatsos, G., 2002. In: Proceedings of the 12th National Conference and Technology Exhibition on Biomass for Energy. Industry and Climate Protection, Amsterdam, pp. 489-493.

Winer, N., 1980. The potencial of the carob (Ceratonia siliqua L). Int. Tree Crops J. 1, 15-26.

Zohary, M., 1973. Geobotanical Foundations of the Middle East, 2 vols. Stuttgart.

Zohary, M., 1983. Wild genetic resources of crops in Israel. Israel J. Bot. 32, 97-127. 